\section{Menisco-ligamentous injuries of knee joint: Can ultrasonogra- phy serve as an effective screening modality?}

\author{
Farooq Mir, ${ }^{1}$ Zahoor Raina, ${ }^{2}$ Omair \\ Shah, ${ }^{2}$ Tariq Gojwari, ${ }^{2}$ Irfan Robbani, ${ }^{2}$ \\ Tahleel Shera $^{2}$ \\ ${ }^{1}$ Department of Radiology, SKIMS \\ Medical College Bemina, J\&K; \\ ${ }^{2}$ Department of Radiology, SKIMS \\ Soura, J\&K, India
}

\begin{abstract}
The aim is to calculate sensitivity, specificity and diagnostic accuracy of Ultrasonography (USG) as a screening modality in evaluation of MeniscoLigamentous injuries of knee joint with Magnetic Resonance Imaging (MRI)as gold standard for comparison.Patients with clinically suspected Menisco-Ligamentous injurieswere evaluated by USG initially followed by MRI on the same day. A total of 60 patients (50 males, 10 females) underwent USG and MRI. USG was done using high frequency probe $(9-14 \mathrm{~Hz})$ and all the injuries noted. USG of the normal knee was done for comparison. MRI with trauma protocol sequences was done on the same day. The accuracy of USG and MRI in diagnosis of menisco-ligamentous injuries was compared.Majority of the patients $(50 \%)$ belonged to age group of 21-40 years. Most common injuries seen were medial meniscal tear followed by medial collateral ligament injury. The strength of agreement between USG and MRI was good with Diagnostic accuracy of USG ranging from $83.3 \%$ to $95 \%$ for different meniscal/ligamentous injuries.USG is an effective imaging modality with high accuracy in diagnosing menisco-ligamentous injuries. USG can act as an effective screening modality in closed knee trauma for evaluation of menisco-ligamentous injuries especially in resource constrained regions owing to its easy availability, portability and lower cost. MRI can be reserved for patients with suspicious USG and clinical findings.
\end{abstract}

\section{Introduction}

The knee joint is a compound synovial joint that consists of hyaline cartilage articulations between femur, tibia and patella. The major stabilizers of the knee joint include its ligamentous structures andinjury to these supporting structures is quite common.Knee injuries are commonly sports related. ${ }^{1}$

The knee joint is stabilized by a number of ligaments. Medially, the medial collateral ligament extends from medial femoral condyle to the tibia in the coronal plane. The lateral or fibular collateral ligament originates from lateral femur and extends over the popliteus tendon to insert on the lateral aspect of fibula with the biceps femoris tendon. The anterior and posterior cruciate ligaments within the intercondylar notch extend from femur to the proximal tibia as intra-capsular but extra-synovial structures. The menisci are C-shaped fibrocartilagenous structures present between femur and tibia acting as shock absorbers. ${ }^{2}$

Clinical evaluation and localization of knee injuries is quite difficult. Imaging plays a pivotal role in management of knee injuries. While radiographs are limited to bony injuries, USG and MRI have been widely used in the past for assessing ligaments, menisci and soft tissue injuries. MRI provides multiplanar imaging capabilities, is non-invasive and lacks radiation. It provides a wide range of information in cases of knee injury ranging from ligamentous injuries to bone and meniscal injury. The disadvantage, if any, is lack of availability, cost of the study and few contraindications like claustrophobia and metallic plates common in trauma patients. ${ }^{3,4}$ Arthroscopy is considered as the gold standard and has the advantage of being both diagnostic and therapeutic. However it is invasive and associated with complications like deep vein thrombosis, pulmonary embolism and infections. ${ }^{5-7}$

High resolution Ultrasound (USG) has emerged in the recent decade as an easily available modality for evaluation of knee trauma. Although the sensitivity and specificity might not be better than MRI, but USG has shown to be comparable to MRI in diagnosing certain injuries including joint effusion, muscle and tendon injury and meniscal injuries. Nerves and vessels around knee joint can also be reliably assessed. The advantages of USG include its availability, possibility of bedside use and use in patients with contraindications to MRI. Comparison of the injured knee with the normal side is also possible. ${ }^{8}$

The role of USG therefore needs to be evaluated vis-a-vis knee injuries. Our study aims at assessing HRUSG as an initial screening modality in patients with menisco-ligimentous injuries of knee and along with clinical examination decide whether further evaluation in the form of MRI and/or arthroscopy is required or not.
Correspondence: Omair Shah, Department of Radiology, SKIMS SOURA, Srinagar J\&K, 190010 India.

Tel.: 91.7006560813

E-mail: shahomair133@gmail.com

Keywords: Magnetic resonance imaging; ultrasonography; menisco-ligamentous injuries; knee joint.

Acknowledgement: Department of Orthopaedics, SKIMS Medical College Bemina, J\&K, India.

Contributions: MF, study design, data collection, statistical analysis, data interpretation, manuscript preparation, literature search; RZ.study design, data collection, data interpretation, literature search; SO. study design, data interpretation, manuscript, preparation; GT. data collection, statistical analysis, manuscript preparation; RI, study design, statistical analysis, data interpretation; ST, data collection, data interpretation, manuscript preparation.

Conflict of interest: The authors have no conflict of interest to declare.

Availability of data and materials: All data generated or analyzed during this study are included in this published article.

Ethics approval and consent to participate: Not applicable.

Informed consent: Written informed consent was obtained from a legally authorized representative(s) for anonymized patient information to be published in this article

Received for publication: 7 March 2021.

Revision received: 8 June 2021.

Accepted for publication: 15 July 2021

This work is licensed under a Creative Commons Attribution 4.0 License (by-nc 4.0)

${ }^{\circ}$ Copyright: the Author(s), 2021

Licensee PAGEPress, Italy

Healthcare in Low-resource Settings 2021; 9:9731 doi:10.4081/hls.2021.9731

\section{Materials and Methods}

The prospective study was conducted at Sher-i-Kashmir Institute of Medical Sciences, Srinagar over a period of two years (2018-2020). Sixty patients clinically suspected of having knee ligamentous or meniscal injuries were included in the study. Patients with poly trauma with hemodynamic instability, open wounds around the knee and those in whom fractures had been fixated were excluded from the study. All the patients underwent USG of the injured knee as well as normal knee using 
GE Logic P5 high resolution USG machine and MR of the injured knee on the same day. Image interpretations were done by radiologists with more than 5 years experience in musculoskeletal radiology. The findings of USG and MRI were kept mutually blinded for the radiologists. All the patients were clinically examined by an orthopaedic with more than 15 years of experience and a clinical diagnosis was made based on various clinical signs which again were not revealed to the radiologist.

\section{Ultrasound technique}

All $\mathrm{USG}^{9,10}$ examinations were performed using LOGIC GE P5 machine equipped with high frequency linear probe $(7-15 \mathrm{~Hz})$. Menisci were examined in partially flexed knee along lateral and medial aspects. Normal meniscus was described as a triangular shaped hyperechoic structure located at the joint space while meniscal tears were suggested byappearance of hypoechoic or anechoic clefts within the structure. PCL was evaluated in prone position from popliteal fossa with the knee fully extended. Normal PCL appeared homogenously hypoechoic with a well defined posterior border, while torn PCL is heterogeneously hypoechoic with increased antero-posterior diameter and/or indistinct or wavy posterior margins.Direct visualization of ACL being extremely difficult, ACL injuries were indirectly diagnosed by haemarthrosisseen as a fluid collection in intercondylar space when knee is examined in full flexion together with anterior translation of knee on dynamic USG. MCL and LCL are examined in semi-flexed (30-50 degrees) position of knee along medial and lateral aspects respectively. The torn MCL was diagnosed in the form of thickening and heterogeneously hypoechoic change of the ligament on sonogram.LCL is usually depicted as a hypoechoic thin band like structure; however its oblique orientation makes assessment difficult owing to anisotropic effect (Figure 1).

\section{MRI technique and protocol}

A careful trace of the ligament from its distal insertion in the fibular head to the proximal femoral insertion was done for complete evaluation. Note was made of any additional feature including effusion, tendon or muscle injury and patellar fracture.MRI was carried out using 1.5T MR system (Magnetom Avanto, Siemens Medical Systems, Erlangen, Germany) using the set protocol in our institution (Table 1)._Meniscal tears on MRI were diagnosed by abnormal meniscal morphology and intra-substance high signal intensity on T2W and PD images. Ligament tears were seen as high signal intensity on $\mathrm{T} 2 \mathrm{~W}$ and PD images and interruption or disruption of fibres.
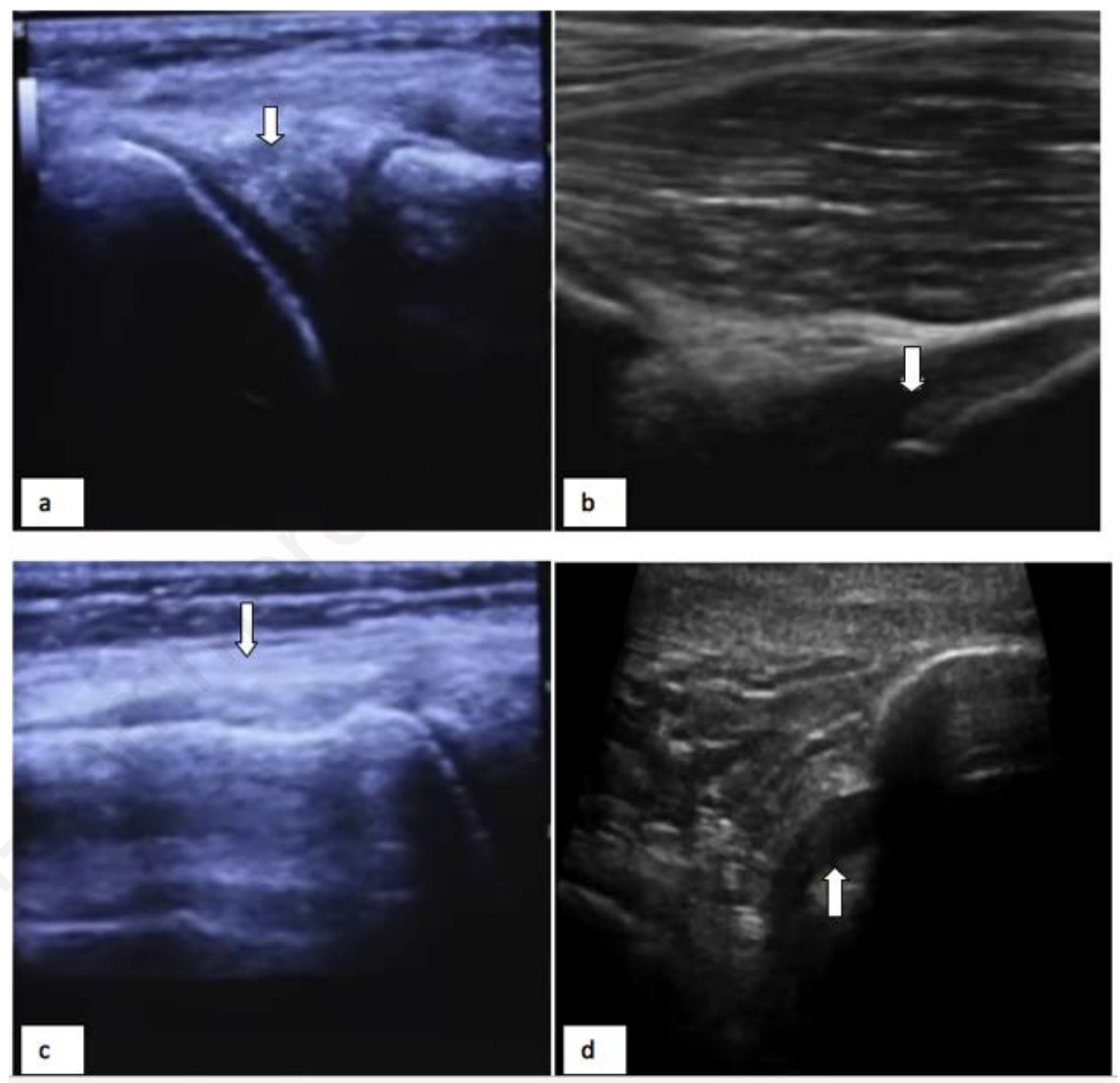

Figure 1. Normal morphology of menisco-ligamentous component of knee joint on USG. a) Medial meniscus; b) Posterior cruciate ligament; c) Medial collateral ligament; d) Anterior cruciate ligament.

Table 1. MRI protocol for knee trauma at our institution.

\begin{tabular}{|c|c|c|c|c|c|c|}
\hline Sequence & TR & $\mathrm{TE}$ & FOV read & FOV phase & Slice thickness & Flip angle \\
\hline T1- Coronal & 3650 & 42 & 160 & 90.6 & $3.5 \mathrm{~mm}$ & 150 \\
\hline T1 -TIRM- coronal & 527 & 12 & 160 & 100 & $3.0 \mathrm{~mm}$ & 150 \\
\hline PD- FS- axial & 3320 & 24 & 150 & 100 & $3.0 \mathrm{~mm}$ & 150 \\
\hline PD-FS- coronal & 3320 & 24 & 150 & 100 & $3.0 \mathrm{~mm}$ & 150 \\
\hline PD -FS -sagittal & 3320 & 24 & 150 & 100 & $3.0 \mathrm{~mm}$ & 150 \\
\hline T2-MEDIC-axial & 623 & 16 & 150 & 100 & $3.0 \mathrm{~mm}$ & 30 \\
\hline
\end{tabular}

PD: Proton Density, FS: Fat Saturated, TIRM: Turbo Inversion Recovery Magnitude, MEDIC: Multiple Echo Data Image Combination. 
/ True Positive + True Negative + False Positive + False Negative).

\section{Results}

\section{Patient profile}

We studied 60 patients suspected of having menisco-ligamentous injuries of knee joint with a meanage of $35 \pm 8$ years (range 17-59 years), majoritybelonging to the age group of 21 to 30 years $(n=37$, $62 \%$ ). Male to female ratio was $4: 1$ and right knee $(\mathrm{n}=45,75 \%)$ was more commonly involved than left knee $(\mathrm{n}=15$, $25 \%)$. Sports related injuries $(n=38,63 \%)$ followed by road traffic accidents $(n=17$, $28 \%$ ) were the main causes of knee joint injuries.

\section{Evaluation of ACL}

Out of 60 cases, $11(18 \%)$ cases were positive by USG and clinical examination. Only 09 of these were proved to be positive on MRI, thusresulting in 09 true positives and 02 false positives.

Out of 60 cases, 49 cases were negative on USG and 46 proved to be negative on MRI resulting in 46 true negatives and 03 false negatives. The clinical examination was negative in 40 cases only thereby over diagnosing ACL tear.

\section{Evaluation of PCL}

PCL injury was diagnosed on USG in $15(25 \%)$ cases. MRI in these cases was suggestive of PCL injury in 7 cases resulting in 7 true positives and 8 false positives.

Out of 60 cases, 45 cases were negative on USG and 43 proved to be negative on MRI resulting in 43 true negatives and 02 false negatives.

\section{Evaluation of menisci}

For Medial meniscal tear, USG was consistent with MRI in $53(88 \%)$ patients consisting of 20 true positives, 33 true negatives, 3 false positives and 4 false negatives.

For Lateral meniscal tear, USG was consistent with MRI in $50(83 \%)$ patients consisting of 5 true positives, 45 true negatives, 06 false positives and 04 false negatives.

All meniscal tears $(n=25)$ that were detected by USG were high grade tears (grade 2 and 3) on MRI (Figure 2)and meniscal tears $(n=8)$ which were missed by USG belonged to grade 1 category on MRI.

\section{Evaluation of collateral ligaments}

MCL injury was seen in 15 (25\%) patients on USG. All of these cases were positive on MRI also resulting in 15 true positives. Out of 45 patients who were negative on USG, only 41patients were negative on MRI resulting in 41 true negatives and 04 false negatives.

LCL injury was seen in $7(12 \%)$ patients on USG, all of which were positive on MRI resulting in 07 true positives. However out of 53 patients who were negative on USG, 50 patients were negative on MRI resulting in 50 true negatives and 03 false negatives.

\section{Discussion}

USG in menisco-ligamentous knee injuries has remained an enigma over many decades. With variable results in many previous studies its role remains ambiguous. Our study aimed at setting
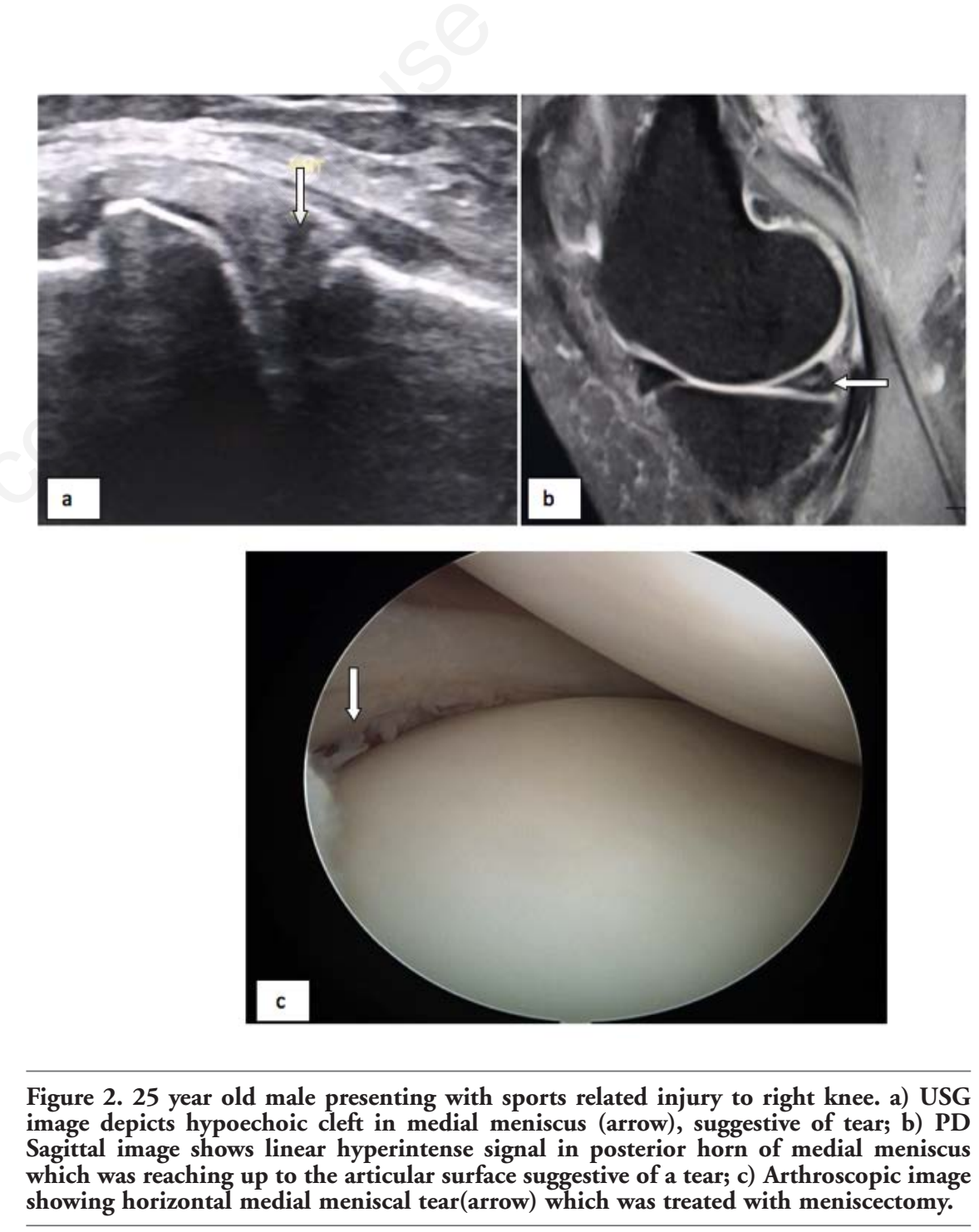

Figure 2. 25 year old male presenting with sports related injury to right knee. a) USG image depicts hypoechoic cleft in medial meniscus (arrow), suggestive of tear; b) PD Sagittal image shows linear hyperintense signal in posterior horn of medial meniscus which was reaching up to the articular surface suggestive of a tear; c) Arthroscopic image showing horizontal medial meniscal tear(arrow) which was treated with meniscectomy.

out clear guidelines vis-a-vis use of USG in knee ligaments and meniscus injuries. We included a total of 60 patients with a mean age of 35.7 years with male predominance $(83 \%)$. The results we obtained were are quite similar to those of Nasir et al. ${ }^{11}$ indicating the increased incidence of knee injuries in young males owing to more participation in contact sports and outdoor work compared to females in our part of the world.

We assessed the accuracy of USG in regards to closed knee injuries with clinical suspicion of menisco-ligamentous injuries taking MRI as the gold standard. We found USG to be consistent with MRI in $55(91.67 \%)$ out of 60 patients. Sensitivity, specificity and accuracy of ultrasound in detecting ACL injury was $75 \%, 95.8 \%$ and $91.67 \%$ respectively. Our results were concordant with the studies done by Friedlet al. ${ }^{12}$ (Sensitivity $70 \%$ and specificity 98\%), Ptaszniket al. ${ }^{13}$ (Sensitivity $91 \%$ and specificity $100 \%$ ) and Monem et al. ${ }^{14}$ (Sensitivity 
$81 \%$ and specificity $84 \%$ ) showing comparable statistics. The lower sensitivity of USG in diagnosing ACL injuries is probably because of dependence on indirect signs for diagnosis and lack of direct visualization. However, indirect signs of ACL tear on USG together with clinical suspicion of ACL injury should serve as an indication for MRI of the injured knee (Figure 3). For Posterior Cruciate Ligament tears (Figure 4), ultrasound was consistent with MRI in $50(83.3 \%)$ out of 60 patients having a sensitivity, specificity and accuracy of $77.7 \%, 84.3 \%$ and $83.3 \%$ respectively. The diagnostic accuracy of our study vis-a-vis PCL tears was comparable to previous studies conducted by Wang et al. ${ }^{15}$ (Sensitivity $83.3 \%$, specificity $87.0 \%$ and an accuracy of $85.7 \%$ ) and Bhanupriya et al. ${ }^{16}$ (Sensitivity $75 \%$, specificity $93 \%$ and accuracy $92 \%$ ). The overall diagnostic accuracy of USG in cruciate ligament injury can be regarded as acceptable and in resource constrained regions like ours, USG together with clinical examination can help guide MRI examinations. Also the injuries which remained elusive on USG were mostly grade 1 and 2 injuries which are otherwise also managed conservatively.

The menisci are important components of knee joint function. Tears of menisci can be assessed on HRUSG and appears in the form of hypoechoic area within the meniscus substance. USG in our study helped in the diagnosis of medial meniscal tears with a sensitivity, specificity and accuracy of $83.3 \%, 91.67 \%$ and $88.3 \%$ respectively. Lateral meniscal tears on the other hand had a sensitivity, specificity and accuracy of $55.5 \%$, $88.23 \%$ and $88.33 \%$ respectively. The results match those described by Monem et al., ${ }^{14}$ Bhanupriyaet al. ${ }^{16}$ and Attya. ${ }^{17}$ Bhanupriya et al. ${ }^{16}$ demonstrated sensitivity, specificity and accuracy of $83.8 \%$, $89.4 \%$ and $86 \%$ for medial meniscal tears and $40 \%, 91 \%$ and $78.3 \%$ for lateral meniscal tears. The diagnostic accuracy for lateral and medial meniscal tear assessment by USG in the study by Monem et al. ${ }^{14}$ was $83 \%$ and $76 \%$ while that of Attya. ${ }^{17}$ was $88 \%$ and $73 \%$ respectively. Majority of the meniscal tears detected by USG in our study were high grade tears on MRI and all located in peripheral red zone or red-white zone of the meniscus. The other associated finding we observed were para-meniscal cysts seen in $10(30 \%)$ of our patients with meniscal tear $(n=33)$. The disadvantages were lack of classification of meniscal tears and inability to identify displaced meniscal fragments as well as variants like discoid meniscus, which can be a predisposing factor for tears. The classification of meniscal tears was well established on the MRI with 17(52\%) horizontal type, $10(30 \%)$ radial tears, $4(12 \%)$ bucket handle type and $2(6 \%)$ flap type
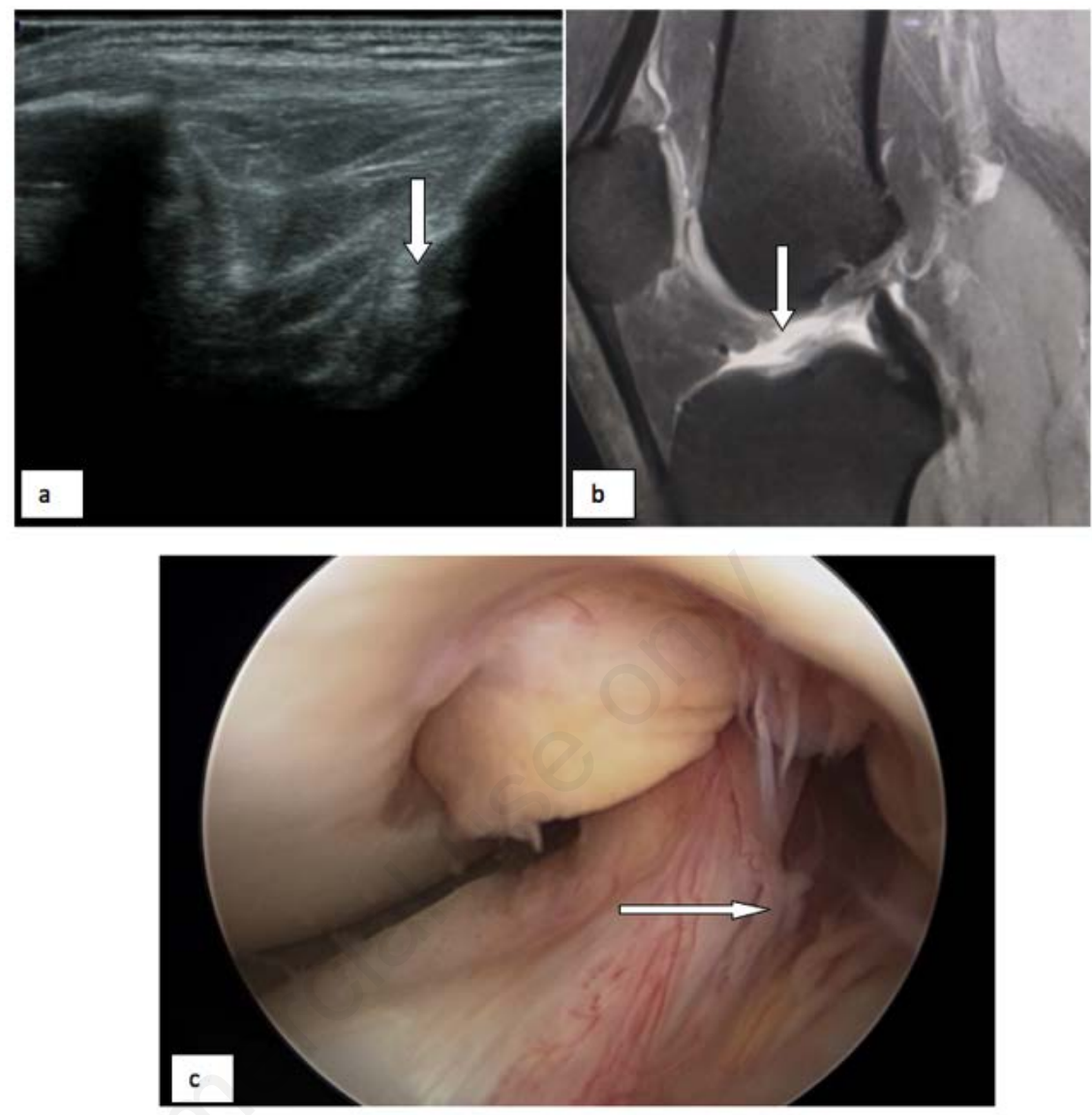

Figure 3. 38 year old female with history of injury to left knee.a) Longitudinal USG imagedepicts disruption of anterior fibres (arrow) of ACL; b)PD Sagittal imageshowing hyperintense signal involving ACL with disruption of fibres. Some fibres are seen posteriorly suggesting a near total tear; c) Arthroscopic imageshowing partial ACL tear (arrow) which was repaired with an ACL graft.

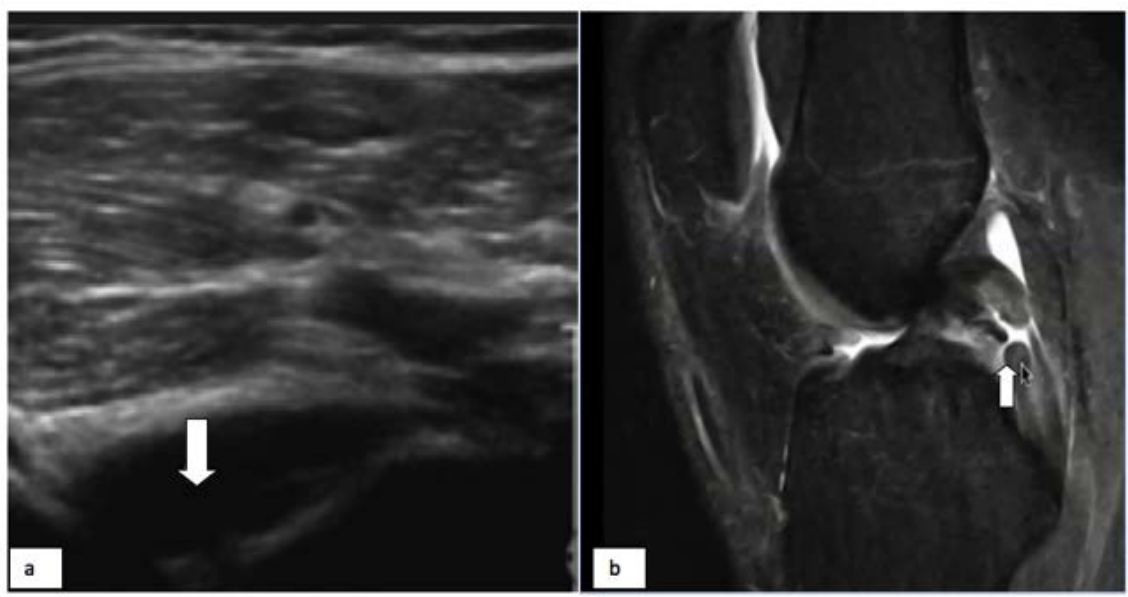

Figure 4. 40 year old male with injury to left knee. a) USG image depicts thickened hypoechoic PCL suggestive of PCL injury. Fibre continuity could not be assessed; b) PD Sagittal image depicts hyperintense signal with associated fibre disruption suggestive of a complete PCL tear. Joint effusion is also noted. 


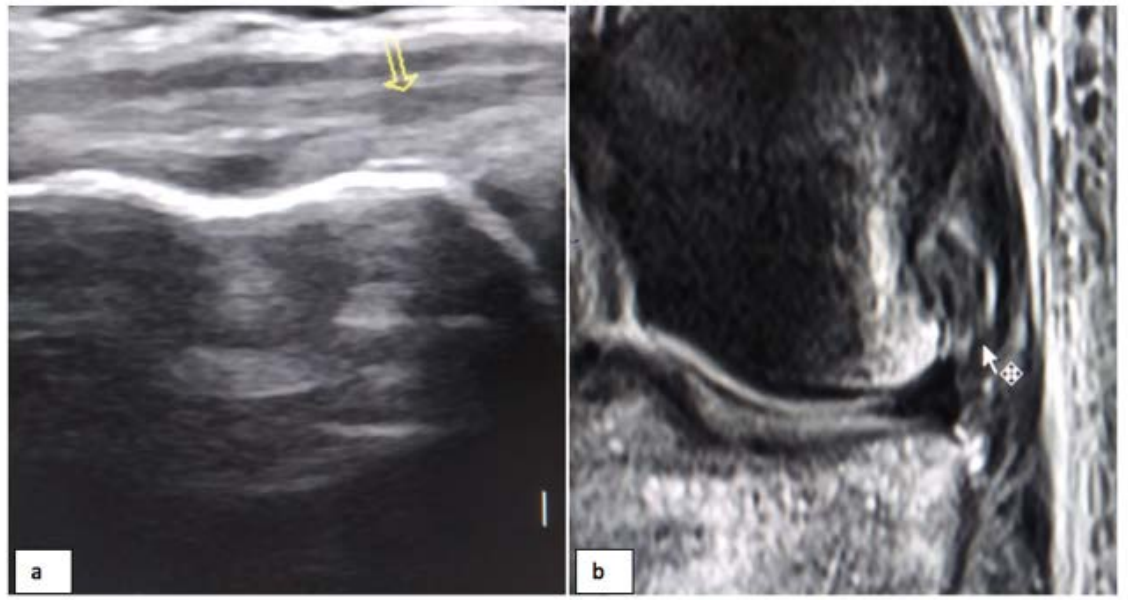

Figure 5. 22 year old male with injury to right knee. a) Longitudinal USG image showing mildly bulky hypochoic MCL (yellow arrow) with no frank disruption of fibers suggestive of MCL sprain; b) PD coronal image depicts hyperintensesignal in the bulky MCL with maintained fiber continuity (cursor). Contusions involving the medial femoral and tibial condyles are also noted.

tears. The predominance of medial meniscus tears is probably due to more sports related injuries in our study especially the twist injuries owing to the bad quality grounds in our part of the world. The role of USG in meniscal tears can be termed as an initial imaging modality owing to its easy availability and ready use in any environment. Also meniscal tears detected on USG tend to be major tears in nature and hence arthroscopy can be directly advised to confirm the diagnosis and therapeutic intervention in the same go. However in USG negative cases with strong suspicion of meniscal tear, a MRI should be the answer.

The collateral ligaments are well visualized on USG owing to their superficial location and are lateral stabilizers of the knee joint (Figure 1). In our study, the sensitivity, specificity and accuracy of ultrasound for detection of MCL injury was $78.9 \%, 100 \%$ and $93.3 \%$ and for LCL injury was $70 \%, 100 \%$ and $95 \%$ respectively. Our results are corroborated by the study done by Amandeep et al. ${ }^{18}$ who observed a sensitivity, specificity and accuracy of $84.6 \%, 100 \%$ and $96.6 \%$ for MCL injury and $84.6 \%, 97.8 \%$ and $95 \%$ for LCL injury. Bhanupriya et al. ${ }^{16}$ recorded diagnostic accuracies of $96 \%$ and $94 \%$ for MCL and LCL respectively. The high specificity and diagnostic accuracy makes USG a potent imaging modality as far as collateral ligament injury is concerned (Figure 5). The increased number of MCL tears $(n=15)$ in comparison to LCL $(n=7)$ again goes with increased sports related injuries in our study group as a part of the unhappy triad (medial meniscus tear + medial collateral ligament tear + ACL tear). Therefore a MCL injury on USG should prompt the radiologist to look for other injuries and thereby guide management.

The limitations of the study included the lesser number of patients probably owing to the current COVID-19 pandemic. A larger study is warranted to obtain further statistical correlations. Arthroscopy was not done in all patients and hence confirmation of MRI and USG findings were not possible. However, considering high diagnostic accuracy of MRI, role of USG can be assessed based on MRI findings as standard.

\section{Conclusions}

USG is an effective screening modality for menisco-ligamentous injuries of the knee. Collateral ligament injuries can be completely assessed by USG. Major meniscal and cruciate ligament tears can be diagnosed on USG, however grades 1 and 2 injuries, deep injuries and classification is not possible.Indirect signs like haemarthosis, parameniscal cysts and muscle/tendon tears together with clinical suspicion can serve as guides for further imaging in the form of MRI. A wide availability, lower cost and fair reliability makes USG a modality of first choice for evaluation of knee injuries in resource constrained countries with MRI being reserved for patients with suspicious USG results.

\section{References}

1. Kapur S, Wissman RD, Robertson M, et al. Acute knee dislocation: revive of an elusive entity. Curr Probl Diagn Radiol 2009;38:237-50.

2. Abulhasan JF, Grey MJ. Anatomy and physiology of knee stability. J Funct Morphol Kinesiol 2017;2:34.

3. Yaqoob J, Alam MS, Khalid N. Diagnostic accuracy of magnetic resonance imaging in assessment of meniscal and ACL tear: Correlation with arthroscopy. Pak J Med Sci 2015;31:263-8.

4. Navali AM, Bazavar M, Mohseni MA, et al. Arthroscopicevaluation of the accuracy of clinicalexamination versus MRI in diagnosingmeniscustears and cruciate ligamentruptures. Arch Iran Med 2013;16:229-32.

5. Ward BD, Lubowitz JH. Basic knee arthroscopy part 3: Diagnostic arthroscopy. Arthrosc Tech 2013;2:5035.

6. Friberger Pajalic K, Turkiewicz A, Englund M. Update on the risks of complications after knee arthroscopy. BMC Musculoskelet Disord 2018;19:179.

7. Salzler MJ, Lin A, Miller CD, et al. Complications after arthroscopic knee surgery. Am J Sports Med 2014;42:2926.

8. Jacobson JA. Knee ultrasound. In: Jacobson JA, ed. Fundamentals of musculoskeletal ultrasound, 2nd edition. Philadelphia, PA: Elsevier Saunders; 2013: 212-56.

9. Patil P, Dasgupta B. Role of diagnostic ultrasound in the assessment of musculoskeletal diseases. Ther Adv Musculoskelet Dis 2012;4:341-55.

10. Bianchi S, Martinoli C, Bianchi S. Knee. In: Baert AL, ed. Ultrasound of the musculoskeletal system. Berlin Heidelberg: Springer-Verlag; 2007: 637-744.

11. Nasir AI.The role of magnetic resonance imaging in the knee joint injuries. Int Res J Medical Sci2013;1:1-7.

12. Friedl W, Glaser F. Dynamicsonography in the diagnosis of ligament and meniscal injuries of knee.Arch Orthop Trauma Surg 1991;110:132-8.

13. Ptasznik R, Feller J,Bartlett J, et al.The value of sonography in the diagnosis of traumatic rupture of the anterior cruciate ligament of the knee. Am J Roentgenol 1995;164:1461-3.

14. Monem SAE, Enaba MM. Comparative study between High Resolution Ultrasound (HRUS) and MRI in the diagnosis of meniscal and cruciate ligament injury of the knee. Med J Cairo 
Univ2012; 233-42.

15. Wang C, Shih T, Wang H, Chiu Y, Wang

T. The accuracy of ultrasonographic examination of injured posterior cruciate ligament. J Med Ultrasound 2009; 17:187-92.
16. Bhanupriya S, Khushal N, Suhas SG, et al. Evaluationof knee joint by USG And MRI. IOSR-JDMS 2016;15:122-31.

17. Attya MSA. Evaluation of role of non ionized radiology tools in knee soft tissue injuries. Al-Azhar Assiut Med J
2015;13:52-9.

18. Amandeep S, Indermeet $M$, Thukral CL, et al. Diagnostic accuracy of USG in Evaluation of knee injuries with MRI correlation. IJARS 2018;7:RO50-5. 